\title{
Low levels of soluble TWEAK, indicating on- going inflammation, were associated with depression in type 1 diabetes: a cross- sectional study
}

Eva O. Melin ${ }^{1,2^{*}}$, Jonatan Dereke ${ }^{1}$ and Magnus Hillman ${ }^{1}$

\begin{abstract}
Background: Low levels of the soluble tumour necrosis factor-like weak inducer of apoptosis (STWEAK) and depression are linked to cardiovascular disease. Galectin-3, inadequate glycemic control and low high-density lipoprotein (HDL)-cholesterol levels were previously linked to depression in these patients with type 1 diabetes mellitus (T1DM). The main aim was to explore whether sTWEAK was associated with depression. A secondary aim was to explore diabetes related variables associated with low STWEAK.

Methods: Cross-sectional design. T1DM patients ( $n=283$, men 56\%, age18-59 years) were consecutively recruited from one specialist diabetes clinic. Depression was defined as Hospital Anxiety and Depression Scale-Depression sub scale $\geq 8$ points. Blood samples, anthropometrics and blood pressure were collected, supplemented with data from electronic health records. Enzyme linked immunosorbent assays were used to measure sTWEAK and galectin3. Low sTWEAK was defined as $<7.2 \mathrm{ng} / \mathrm{ml}$ and high galectin-3 as $\geq 2.6 \mathrm{ng} / \mathrm{ml}$. Multiple logistic regression analyses were performed, calibrated and validated for goodness of fit. We adjusted for age, sex, diabetes duration, galectin-3, metabolic variables, serum-creatinine, smoking, physical inactivity, medication, and cardiovascular complications.

Results: For 29 depressed versus 254 non-depressed patients the prevalence rates were for low STWEAK: 93 and 68\% ( $p=0.003)$ and for high galectin-3: 34 and 13\% ( $p=0.005)$ respectively. HDL-cholesterol levels were lower for the depressed $(p=0.015)$. Patients with low sTWEAK versus high sTWEAK had lower usage of continuous subcutaneous insulin infusion (CSII) (6\% versus 17\%, $p=0.005)$.

Low sTWEAK (adjusted odds ratio (AOR) 9.0, $p=0.006$ ), high galectin-3 (AOR 6.3, $p=0.001$ ), HDL-cholesterol (per $\mathrm{mmol} / \mathrm{I}$ ) (AOR 0.1, $p=0.006$ ), use of antidepressants (AOR 8.4, $p<0.001$ ), and age (per year) (AOR 1.05, $p=0.027$ ) were associated with depression. CSII (AOR 0.3, $p=0.003$ ) and depression (AOR 7.1, $p=0.009$ ) were associated with low sTWEAK.

(Continued on next page)
\end{abstract}

\footnotetext{
* Correspondence: eva.melin@kronoberg.se

${ }^{1}$ Lund University, Faculty of Medicine, Clinical Sciences, Diabetes Research Laboratory, Lund, Sweden

2Department of Research and Development, Region Kronoberg, Box 1223,

SE-351 12 Växjö, Sweden
}

(c) The Author(s). 2020 Open Access This article is licensed under a Creative Commons Attribution 4.0 International License, which permits use, sharing, adaptation, distribution and reproduction in any medium or format, as long as you give appropriate credit to the original author(s) and the source, provide a link to the Creative Commons licence, and indicate if changes were made. The images or other third party material in this article are included in the article's Creative Commons licence, unless indicated otherwise in a credit line to the material. If material is not included in the article's Creative Commons licence and your intended use is not permitted by statutory regulation or exceeds the permitted use, you will need to obtain permission directly from the copyright holder. To view a copy of this licence, visit http://creativecommons.org/licenses/by/4.0/. The Creative Commons Public Domain Dedication waiver (http://creativecommons.org/publicdomain/zero/1.0/) applies to the data made available in this article, unless otherwise stated in a credit line to the data. 
(Continued from previous page)

Conclusions: Lower levels of sTWEAK and HDL-cholesterol and higher levels of galectin-3 were independently associated with depression in T1DM. These factors might all contribute to the increased risk for cardiovascular disease and mortality previously demonstrated in patients with depression. CSII (inversely) and depression were independently associated with low sTWEAK levels.

Keywords: Cardiovascular complications, Continuous subcutaneous insulin infusion, Depression, Galectin-3, HbA1c, High-density lipoprotein-cholesterol, Inflammation, Soluble tumour necrosis factor-like weak inducer of apoptosis, Type 1 diabetes mellitus

\section{Background}

Type 1 diabetes (T1DM) is an autoimmune disease, characterized by insulin deficiency due to pancreatic beta cell loss leading to hyperglycaemia [1]. The introduction of intensive insulin therapy for patients with T1DM has resulted in an increased prevalence of the components of the metabolic syndrome, contributing to an amplified prevalence of cardiovascular complications [2]. Inflammatory disturbances also contribute to cardiovascular disease [3].

In patients with diabetes, depression is associated with increased prevalence of all diabetes-related complications [4], and with increased cardiovascular and allcause mortality [5]. Depression has been linked to metabolic, autonomic and hypothalamic-pituitary-adrenal (HPA)-axis dysregulations with subsequent disturbances of cortisol secretion [6, 7], and there is growing evidence that immuno-inflammatory changes contribute to the development of depression [6-10]. Insulin deficiency, hyperglycemia as well as episodes of hypoglycemia all have impact on the brain, and might contribute to the development of depression in patients with T1DM [7].

The tumour necrosis factor-like weak inducer of apoptosis (TWEAK) is a transmembrane protein, which is a member of the tumour necrosis factor (TNF)-receptor super family [11]. TWEAK is proteolytically processed by furin which leads to the release of soluble (s)TWEAK [11]. In the reverse process, sTWEAK binds to the functional receptor of TWEAK, Fn14 [12]. The receptor Fn14 is highly upregulated in systemic inflammatory states, which leads to increased sTWEAK binding and subsequently lower levels of sTWEAK [12]. The TWEAK/Fn14 axis plays a beneficial role in tissue repair after acute injury. However, it has been shown that chronic TWEAK/Fn14 axis activation is implicated in the development of cardiovascular disease [12]. High levels of sTWEAK are released by normal arteries, but are diminished in people with chronic vascular damage such as carotid stenosis, coronary artery disease and heart failure $[12,13]$, resulting in an increased risk for cardiovascular mortality [14]. Proinflammatory effects of TWEAK on astrocytes in vitro implies that TWEAK could play a significant role in brain inflammation [15]. Low sTWEAK levels have been demonstrated in people with T1DM [16], type 2 diabetes mellitus (T2DM) [17], and gestational diabetes [18]. Low sTWEAK levels have also been demonstrated in depressed people without diabetes [19], and in people with bipolar disorder during ongoing manic episodes [20]. Galectin-3 is a beta-galactoside-binding lectin involved in several inflammatory processes [21]. Increased galectin-3 levels have been linked to coronary artery disease $[22,23]$, heart failure $[24,25]$, prolonged inflammatory responses in the brain [26], and to cardiovascular and allcause mortality $[25,27]$. Systemic inflammation causes decreased high-density lipoprotein (HDL)-cholesterol levels which contribute both to reduced capacity for reverse cholesterol transport, and to reduced capacity to protect lowdensity lipoprotein (LDL)-cholesterol from oxidation [28]. Low HDL-cholesterol levels were previously associated with high levels of galectin-3 binding protein in these patients [29]. Inadequate glycemic control contributes to increased cardiovascular and all-cause mortality [30].

Systematic exploration of these T1DM patients showed that self-reported depression was associated with inadequate glycemic control [31], midnight salivary cortisol secretion [32], galectin-3 [33], and inversely with HDLcholesterol [34]. Depression was not associated with sex, obesity, blood pressure, total cholesterol, LDL-cholesterol, triglycerides, antihypertensive drugs, lipid-lowering drugs, physical inactivity or smoking habits [34]. Neither was depression associated with the inflammatory variables galectin-3 binding protein [29], soluble sCD163 [35], the soluble receptor for advanced glycation end products (sRAGE), nor the extracellular newly identified receptor for advanced glycation end products (EN-RAGE) [36].

We hypothesised that one biological link between depression and cardiovascular complications in depressed patients with T1DM is a chronic inflammatory state due to TWEAK activation with subsequent low levels of sTWEAK. The main aim was to explore whether low levels of sTWEAK were associated with depression in T1DM patients. A secondary aim was to explore diabetes related variables associated with low sTWEAK.

\section{Methods}

Participants and study design

The study has a cross sectional design and included 287 patients with T1DM. For inclusion and exclusion 
criteria, included and missing variables, see Fig. 1. Inclusion criteria were T1DM with $\geq 1$-year duration, in patients 18-59 years of age. Exclusion criteria were pregnancy, severe somatic and psychiatric disorders such as cancer, hepatic failure, end-stage renal disease, Cushing's disease, severe autoimmune disorders such as systemic lupus erythematosus, psychotic disorders, bipolar disorder, severe personality disorders, severe substance abuse, cognitive deficiency (due to stroke, dementia or mental retardation), or inadequate knowledge of the Swedish language [29, 31-36]. As patients with these disorders were excluded, no specific medications for any of these disorders were used by the included patients.
Patients using systemic corticosteroids were excluded [36].

The patients were consecutively recruited from the largest of two specialist diabetes hospital outpatient clinics where all adult patients with T1DM are treated in Region Kronoberg, Sweden [29, 31-36]. The patients who attend the clinic every 6 months for regular follow-up visits were consecutively recruited by specialist diabetes physicians or diabetes nurses during a nine-month period, 25 March 2009 to 28 December 2009 [29, 31-36]. The catchment population was 125,000 . A questionnaire was used to assess self-reported depression. Blood samples, anthropometrics and blood pressure were collected, supplemented

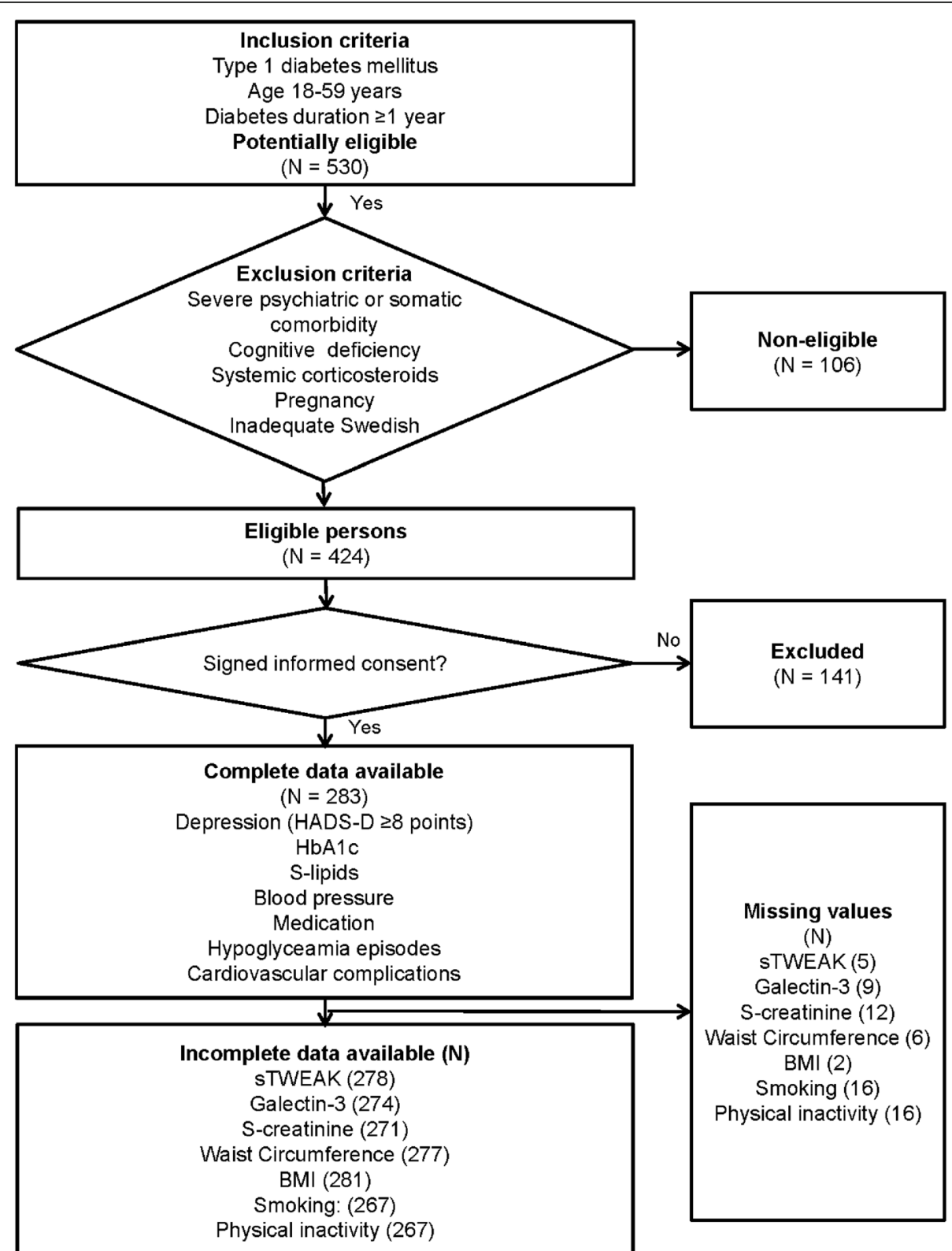

Fig. 1 Flow chart describing the included T1DM patients and included variables in this study of depression 
with data from electronic medical records. Data collection was performed at baseline of a randomized controlled study (RCT) for patients with diabetes, Hemoglobin A1c (HbA1c) $>70 \mathrm{mmol} / \mathrm{mol}$ and psychological symptoms (ClinicalTrials.gov: NCT01714986) [37], and the study is one out of several baseline analyses [29, 31-36]. We adjusted for age, sex, diabetes duration, galectin-3, metabolic variables, serum (s)-creatinine, smoking, physical inactivity, medication, and cardiovascular complications.

\section{Self-reported depression}

Depressive symptoms were assessed by the Hospital Anxiety and Depression Scale - the depression subscale (HADS-D), which consists of 7 statements. Each statement has four response alternatives with scores from 0 to 3 . The recommended cut-off level was used to define depression: $\geq 8$ points as in our previous studies [29, 31-36, 38]. HADS was developed to detect symptoms of depression and anxiety in patients repeatedly searching medical care for somatic complaints, where no somatic disorders were confirmed that could explain their symptoms [38]. No higher levels of emotional awareness are necessary to respond to the statements. A major characteristic of HADS-D is that potential symptoms of somatic disease are not included [38]. According to previous research HADS-D is a useful instrument for detecting symptoms of depression, both at an individual and a collective level, and has been demonstrated to have a good reliability and discriminant validity [39].

\section{Biochemical analyses}

Plasma levels of sTWEAK and galectin-3 were measured using commercially available DuoSet enzyme linked immunosorbent assays (ELISA) kits (R\&D Systems, Minneapolis, Mn, USA) and optimised for human plasma. The analyses were run according to the manufacturer's instructions. The samples were diluted $1: 5$ and $1: 2$, and the intra-assay coefficients of variation were 1.8 and $4.3 \%$ respectively for sTWEAK and galectin-3. All samples were run as duplicates. High galectin-3 levels were defined as $\geq 2.6 \mathrm{ng} / \mathrm{ml}$ as in our previous research [33].

HbA1c and serum-lipids were collected after an overnight fast and they were analysed with an Olympus automated clinical chemistry analyser (Olympus $\mathrm{AU}^{\circ}$, Tokyo, Japan). (corresponding to the 72nd percentile) as in our previous research [31]. High HbAlc levels were defined as $>70 \mathrm{mmol} / \mathrm{mol}$ (NGSP $>8.6 \%$ ). The intra-assay coefficients of variation were for HbA1c $<1.2 \%$; total cholesterol < 2.1\%; HDL-cholesterol < 3.0\%; LDL-cholesterol < $2,6 \%$; and for triglycerides $<2.2 \%$.

S-creatinine was assayed by an $\mathrm{AU} 2700^{\circ}$ instrument (Beckman Coulter, Brea, Ca, USA). The intra-assay coefficient of variation was $<3 \%$.

\section{Anthropometrics and blood pressure}

Waist circumference (WC), weight, length and blood pressure were measured according to standard procedures by a nurse. Body Mass Index (BMI) $\left(\mathrm{kg} / \mathrm{m}^{2}\right)$ was calculated. Abdominal obesity was defined as $\mathrm{WC} \geq 1.02$ $\mathrm{m}$ for men and as $\mathrm{WC} \geq 0.88 \mathrm{~m}$ for women as in our previous studies [29, 31-36, 40].

\section{Insulin resistance}

A ratio between triglycerides and HDL-cholesterol was calculated for the estimation of insulin resistance [41].

\section{Episodes of hypoglycemia}

A severe episode of hypoglycemia was defined as hypoglycemia to such a degree that the patient needed help from another person. Episodes during the last 6 months prior to recruitment were registered [29, 31-36].

\section{Smoking and physical inactivity}

Smokers were defined as having smoked any amount of tobacco during the last year. Physical activity was dichotomized into physical inactivity which was defined as less than 30 min of moderate activities once a week, and physical activity which represents all other levels of physical activity $[29,31-36]$.

\section{Cardiovascular complications}

Cardiovascular complications were defined as ischemic heart disease, cardiac failure, stroke, or transient ischemic attack [29, 31-36].

\section{Diabetes medication}

Patients used either multiple daily insulin injections (MDII) or continuous subcutaneous insulin infusion (CSII) [29, 31-36].

\section{Antidepressants}

Antidepressants were SSRIs (ATC codes N06AB04 or N06AB10); SNRIs (ATC code N06AX16); combined serotonin and norepinephrine reuptake inhibitors (ATC code N06AX21); tricyclic antidepressants (ATC code N06AA04); and/or tetracyclic antidepressants (ATC code N06AX11). The use of antidepressants was dichotomized into users and non-users [29, 31-36].

\section{Lipid-lowering drugs and indications for treatment of hyperlipidemia}

Lipid-lowering drugs were hydroxy-methylglutaryl coenzyme A (HMG-CoA)-reductase inhibitors (statins), (ATC-code C10AA). The use of lipid-lowering drugs was dichotomized into users and non-users of lipidlowering drugs [29, 31-36].

Indications for lipid-lowering drugs were $\mathrm{TC}>4.5$ $\mathrm{mmol} / \mathrm{l} \quad(>1.74 \mathrm{mg} / \mathrm{dl})$ and/or LDL-cholesterol $>2.5$ 
$\mathrm{mmol} / \mathrm{l}$ (>97 mg/dl) according to the Swedish national guidelines in 2009 [42].

\section{Antihypertensive drugs and indications for treatment of hypertension}

Antihypertensive drugs included calcium antagonists (ATC codes C08CA01-02); angiotensin-converting enzyme (ACE) inhibitors (ATC codes C09AA-BA); angiotensin II antagonists (ATC codes C09CA-DA); diuretics (ATC codes C03AA03 or C03CA01); and/or selective beta-adrenoreceptor antagonists (ATC code C07AB). The use of antihypertensive drugs was dichotomized into users and non-users of antihypertensive drugs [29, 31-36].

Indications for antihypertensive drugs were systolic blood pressure $>130 \mathrm{mmHg}$ and/or diastolic blood pressure $>80 \mathrm{mmHg}$ according to the Swedish national guidelines in 2009 [42].

\section{Statistical analysis}

Analysis of data distribution using histograms revealed that age, diabetes duration, sTWEAK, galectin-3, triglycerides, systolic and diastolic blood pressure, were not normally distributed. Data were presented as median (quartile $(\mathrm{q})_{1}, \mathrm{q}_{3}$ ), and analyses were performed with Mann-Whitney $U$ test. Fisher's Exact Test (two-tailed) was used to analyse categorical data, and data were presented as $\mathrm{N}(\%)$. The 60th, 65th, 70th and 75th percentiles of sTWEAK and log-transformed sTWEAK (Lg10) were tried against depression in a backward elimination multiple logistic regression analysis, and the percentile with the highest association was defined as low sTWEAK and was therefore used in the further analyses. Crude odds ratios (CORs) for the associations with depression and with low sTWEAK $(<7.2 \mathrm{ng} / \mathrm{ml})$ were calculated for all included variables. Variables with $p<0.10$ for the CORs were entered into multiple logistic regression analyses (Backward: Wald) with depression and low sTWEAK as dependent variables [43]. The Hosmer and Lemeshow test for goodness-of-fit and Nagelkerke $R^{2}$ were used to evaluate each multiple logistic regression analysis model. Confidence intervals (CIs) of 95\% were used. $P<0.05$ was considered statistically significant. SPSS $^{\oplus}$ version 25 (IBM, Chicago, Il, USA) was used.

\section{Results}

In this study 283 patients with T1DM (56\% men), 1859 years old, diabetes duration 1-55 years were included. Baseline characteristics and laboratory results are compared between 29 patients with depression and 254 patients without depression in Table 1. All patients used either MDII (91\%) or CSII (9\%). The depressed patients had lower median sTWEAK $(p=0.017)$ and median cholesterol $(p=0.044)$; and had higher prevalence of high galectin-3 $(p=0.005)$, high HbA1c $(p=0.026)$, use of antidepressants $(p<0.001)$, and cardiovascular complications $(p=0.012)$.

Associations with depression are presented for $\log$ transformed sTWEAK and four levels of low sTWEAK, representing the 60th, 65th, 70th, and 75th percentiles of sTWEAK, are presented in Table 2. sTWEAK $<7.2$ $\mathrm{ng} / \mathrm{ml}(<70$ th percentile) showed the highest association with depression (adjusted odds ratio (AOR) 6.5, $p=$ 0.010) compared to the 60th, 65th, 75th percentiles and log transformed sTWEAK (all $p$-values $>0.48$ for the AORs).

In Table 3 associations with depression are presented. Age (per year) (AOR 1.05, $p=0.027)$, low sTWEAK (< $7.2 \mathrm{ng} / \mathrm{ml}$ ) (AOR 9.0, $p=0.006$ ), high galectin-3 (AOR $6.3, p=0.001$ ), HDL-cholesterol (per $\mathrm{mmol} / \mathrm{l}$ ) (inversely) (AOR 0.1, $p=0.006$ ), and use of antidepressants (AOR $8.4, p<0.001)$, were associated with depression.

Comparisons between patients with low sTWEAK levels $(<7.2 \mathrm{ng} / \mathrm{ml})$, and high sTWEAK levels $(\geq 7.2 \mathrm{ng} /$ $\mathrm{ml}$ ) are presented in Table 4 . Fourteen percent of the patients with low sTWEAK were depressed and $2 \%$ of the patients with high sTWEAK were depressed ( $p=$ 0.003). Patients with low sTWEAK used CSII (6\%) to a lower extent than patients with high sTWEAK (17\%) $(p=0.005)$.

Associations with low sTWEAK levels are presented in Table 5. Depression (AOR 7.1, $p=0.009$ ) and the use of CSII (inversely) (AOR 0.3, $p=0.003$ ) were independently associated with low sTWEAK $(<7.2 \mathrm{ng} / \mathrm{ml})$.

\section{Discussion}

The main finding of this study of adult patients with T1DM was that the depressed patients had lower levels of sTWEAK than the non-depressed patients. Lower levels of sTWEAK and HDL-cholesterol, higher levels of galectin-3, the use of antidepressants, and age were independently associated with depression. The depressed patients had also higher levels of HbA1c, but the association between high HbA1c and depression was not independent in this context. The use of CSII was inversely associated with low sTWEAK.

Depression is a serious disease with somatic implications, including cardiovascular disease and all-cause mortality [4-7]. Three independent risk factors or risk markers for cardiovascular disease were demonstrated in these depressed patients with T1DM. Low sTWEAK levels [12-14] and high galectin-3 levels [22-25, 27] have previously been linked to the development of cardiovascular disease and increased mortality. HDL-cholesterol levels decrease in inflammatory states [28, 29]. HDL-cholesterol is an established risk marker for cardiovascular disease, but the role of HDL-cholesterol as a causal factor in cardiovascular disease is disputed [44]. In previous research, we have not demonstrated any higher prevalence of 
Table 1 Baseline characteristics, laboratory results, and comparisons between 29 depressed and 254 non-depressed patients with T1DM

\begin{tabular}{|c|c|c|c|c|}
\hline & \multirow[b]{2}{*}{ All } & \multicolumn{3}{|l|}{ Depression } \\
\hline & & Yes & No & $P$ - value ${ }^{\circ}$ \\
\hline$N$ & 283 & 29 & 254 & \\
\hline Age (years) & $(18-59)$ & $49(38,53)$ & $42(31,50)$ & 0.036 \\
\hline Diabetes duration (years) & $(1-55)$ & $21(11,35)$ & $20(10,29)$ & 0.45 \\
\hline \multicolumn{5}{|l|}{ Sex } \\
\hline Women & $124(44)$ & $13(45)$ & $111(44)$ & \multirow[t]{2}{*}{$>0.99^{b}$} \\
\hline Men & $159(56)$ & $16(55)$ & $143(56)$ & \\
\hline Depression & $29(10)$ & - & - & - \\
\hline $\operatorname{sTWEAK}(\mathrm{ng} / \mathrm{ml})^{c}$ & $(0.5-1618)$ & $1.5(1.2,2.9)$ & $2.7(1.3,13.4)$ & 0.017 \\
\hline Galectin-3 (ng/ml) ${ }^{d}$ & $(0.001-100.0)$ & $1.3(0.8,3.0)$ & $0.9(0.5,1.6)$ & 0.011 \\
\hline High galectin-3 $(\geq 2.6 \mathrm{ng} / \mathrm{ml})^{\mathrm{d}}$ & $41(15)$ & $10(34)$ & $31(13)$ & $0.005^{b}$ \\
\hline \multicolumn{5}{|l|}{$\mathrm{HbA1c}$} \\
\hline $\mathrm{mmol} / \mathrm{mol}$ & $(25-110)$ & $68(55,73)$ & $63(54,70)$ & \multirow[t]{2}{*}{0.056} \\
\hline$\%$ & $(4.4-12.2)$ & $8.4(7.2,8.8)$ & $7.9(7.1,8.5)$ & \\
\hline High HbAlc (> $70 \mathrm{mmol} / \mathrm{mol}(>8.6 \%))$ & $75(26)$ & $13(45)$ & $62(24)$ & $0.026^{b}$ \\
\hline Total cholesterol (mmol/l) & $(2.1-10.9)$ & $4.3(4.0,4.8)$ & $4.6(4.1,5.2)$ & 0.044 \\
\hline LDL-cholesterol (mmol/l) & $(0.6-8.3)$ & $2.8(2.2,3.2)$ & $2.8(2.4,3.3)$ & 0.37 \\
\hline Triglycerides $(\mathrm{mmol} / \mathrm{l})$ & $(0.06-5.9)$ & $0.9(0.7,1.5)$ & $0.9(0.7,1.2)$ & 0.35 \\
\hline HDL-cholesterol (mmol/l) & $(0.3-2.7)$ & $1.3(1.2,1.6)$ & $1.6(1.3,1.8)$ & 0.015 \\
\hline Triglycerides/HDL-cholesterol ratio & $(0.04-5.9)$ & $0.6(0.5-1.4)$ & $0.6(0.4-0.9)$ & 0.23 \\
\hline S-Creatinine $(\mu \mathrm{mol} / \mathrm{l})^{\mathrm{e}}$ & $(28-182)$ & $72(63,80)$ & $70(62,78)$ & 0.94 \\
\hline Abdominal obesity ${ }^{f}$ & $46(17)$ & $6(21)$ & $40(16)$ & $0.43^{b}$ \\
\hline BMI $\left(\mathrm{kg} / \mathrm{m}^{2}\right)^{\mathrm{g}}$ & $(17.8-45.2)$ & $23.8(21.8,27.3)$ & $24.8(22.8,27.5)$ & 0.21 \\
\hline Systolic BP (mm Hg) & $(90-160)$ & $120(112,135)$ & $120(110,130)$ & 0.99 \\
\hline Diastolic BP (mm Hg) & $(55-100)$ & $70(68,78)$ & $70(70,75)$ & 0.90 \\
\hline Hypoglycemia (severe episodes) & $12(4)$ & $2(7)$ & $10(4)$ & $0.36^{b}$ \\
\hline Smoking ${ }^{\mathrm{h}}$ & $28(10)$ & $5(18)$ & $23(10)$ & $0.19^{b}$ \\
\hline Physical inactivity ${ }^{i}$ & $29(11)$ & $5(18)$ & $24(10)$ & $0.20^{b}$ \\
\hline Continuous subcutaneous insulin infusion & $25(9)$ & $3(10)$ & $22(9)$ & $0.73^{b}$ \\
\hline Antidepressants & $22(8)$ & $9(31)$ & $13(5)$ & $<0.001^{b}$ \\
\hline Lipid lowering drugs & $130(46)$ & $14(48)$ & $116(46)$ & $>0.84^{b}$ \\
\hline Antihypertensive drugs & $89(31)$ & $10(34)$ & $79(31)$ & $0.68^{b}$ \\
\hline Cardiovascular complications & $10(4)$ & $4(14)$ & $6(2)$ & $0.012^{b}$ \\
\hline
\end{tabular}

Data are presented as (min-max), median $\left(q_{1}, q_{3}\right)$, or $\mathrm{N}(\%)$. ${ }^{a}$ Mann-Whitney $\mathrm{U}$ Test unless otherwise indicated

${ }^{b}$ Fisher's Exact Test. Missing values (N): ${ }^{\mathrm{c}} 5 ;^{\mathrm{d}} \mathrm{g}^{\mathrm{e}}{ }^{\mathrm{e}} 12 ;{ }^{\mathrm{f}} 6^{\mathrm{g}} 2{ }^{\mathrm{h}}{ }^{\mathrm{h}, \mathrm{i}} 16$

metabolic disturbances in the depressed than in the nondepressed, except for increased HbA1c and lower HDLcholesterol in the depressed patients [31, 34]. In this study we added a proxy for insulin resistance [41], which was not associated with depression. Increased HbA1c levels without associated obesity or signs of insulin resistance in the depressed patients could be due to inadequate insulin supply. Insulin deficiency has been suggested as one reason for the development of depression in patients with T1DM [7, 45]. Users of MDII compared to users of CSII had more often low levels of sTWEAK. We haven't found any previous research exploring the associations between sTWEAK and CSII.

One difficulty we had to address was that there is no established consensus regarding normal sTWEAK levels. Therefore, we explored and compared the associations between log transformed sTWEAK, four different definitions of low STWEAK levels and depression. Low sTWEAK levels, defined as levels below the 70th percentile $(<7.2 \mathrm{ng} / \mathrm{ml})$, showed the highest association with 
Table 2 Elimination analyses between log transformed STWEAK, 4 levels of dichotomized sTWEAK and depression

\begin{tabular}{|c|c|c|c|c|c|c|c|c|}
\hline & \multirow[b]{2}{*}{ N (\%) } & \multicolumn{7}{|l|}{ Depression } \\
\hline & & $\begin{array}{l}\text { Yes } \\
\left(\text { Median }\left(q_{1}, q_{3}\right)\right. \\
\text { or } \mathrm{N}(\%))\end{array}$ & $\begin{array}{l}\text { No } \\
\text { (Median }\left(q_{1}, q_{3}\right) \\
\text { or } N(\%))\end{array}$ & $P$ - value ${ }^{a}$ & COR $(95 \% \mathrm{Cl})$ & $P$ - value ${ }^{b}$ & AOR $(95 \% \mathrm{Cl})$ & $P-$ value $^{c}$ \\
\hline sTWEAK (Lg10) & - & $0.2(0.1-0.5)$ & $0.4(0.1-1.1)$ & $0.017^{d}$ & $0.4(0.2-0.9)$ & 0.027 & $1.7(0.4-7.6)$ & 0.48 \\
\hline sTWEAK < $3.9(\mathrm{ng} / \mathrm{ml})$ & $167(60)$ & $24(83)$ & $143(57)$ & 0.009 & $3.6(1.3-9.6)$ & 0.012 & $1.4(0.4-5.0)$ & 0.61 \\
\hline sTWEAK < $6.1(\mathrm{ng} / \mathrm{ml})$ & $181(65)$ & $25(86)$ & $156(63)$ & 0.013 & $3.7(1.3-11.0)$ & 0.018 & $0.5(0.04-6.2$ & 0.58 \\
\hline sTWEAK < $7.2(\mathrm{ng} / \mathrm{ml})$ & $195(70)$ & $27(93)$ & $168(68)$ & 0.003 & $6.5(1.5-28.0)$ & 0.012 & $6.5(1.5-28.0)$ & 0.012 \\
\hline sTWEAK < $11.9(\mathrm{ng} / \mathrm{ml})$ & $209(75)$ & $27(93)$ & $182(73)$ & 0.021 & $5.0(1.2-21.5)$ & 0.032 & $0.0(0.0-)$ & $>0.99$ \\
\hline
\end{tabular}

$N=278 .{ }^{a}$ Fisher's Exact Test unless indicated. ${ }^{\mathrm{b}}$ Logistic regression analysis (simple)

${ }^{c}$ Multiple logistic regression analysis (Backward: Wald). ${ }^{\mathrm{d}}$ Mann-Whitney $\mathrm{U}$ test

depression, and this cut-off level was therefore chosen in the further analyses.

To our knowledge, a potential association between sTWEAK and depression hasn't previously been explored in patients with T1DM. Neither has it been explored whether low sTWEAK, high galectin-3, high HbA1c and low HDL-cholesterol were independently associated with depression. We only found one previous study exploring the association between sTWEAK and depression, and that study was performed in a population without diabetes [19]. Their findings of an association between low sTWEAK levels and depression is in accordance with our findings. Another study showed that manic episodes were linked to low levels of

Table 3 Variables associated with depression in patients with T1DM

\begin{tabular}{|c|c|c|c|c|}
\hline & \multicolumn{4}{|l|}{ Depression } \\
\hline & COR $(95 \% \mathrm{Cl})$ & $P$ - value & AOR $(95 \% \mathrm{Cl})$ & $\begin{array}{l}P \text { - } \\
\text { value }\end{array}$ \\
\hline Age (per year) & $1.04(1.00-1.07)$ & 0.046 & $1.05(1.01-1.10)$ & 0.027 \\
\hline Diabetes duration (per year) & $1.01(0.98-1.04)$ & 0.46 & - & - \\
\hline Sex (women) & $1.0(0.5-2.3)$ & 0.91 & - & - \\
\hline Low sTWEAK (<7.2 ng/ml) & $6.5(1.5-28.0)$ & 0.012 & $9.0(1.9-43.2)$ & 0.006 \\
\hline High galectin-3 ( $\geq 2.6$ ng/ml) & $3.6(1.5-8.5)$ & 0.003 & $6.3(2.2-17.8)$ & 0.001 \\
\hline High HbA1c (> 70 mmol/mol (> 8.6\%)) & $2.5(1.1-5.5)$ & 0.021 & $2.4(0.9-6.3)$ & 0.067 \\
\hline Total cholesterol (per mmol/l) & $0.6(0.4-1.0)$ & 0.040 & $0.7(0.4-1.2)$ & 0.19 \\
\hline LDL-cholesterol (per mmol/l) & $0.7(0.4-1.2)$ & 0.23 & - & - \\
\hline Triglycerides (per mmol/l) & $1.3(0.8-2.0)$ & 0.23 & - & - \\
\hline HDL-cholesterol (per mmol/l) & $0.3(0.1-0.8)$ & 0.020 & $0.1(0.03-0.6)$ & 0.006 \\
\hline Triglyceride/HDL-cholesterol ratio (per unit) & $1.5(1.0-2.3)$ & 0.038 & $1.2(0.6-2.4)$ & 0.56 \\
\hline S-Creatinine (per $\mu \mathrm{mol} / \mathrm{l})$ & $1.00(0.98-1.02)$ & 0.72 & - & - \\
\hline Abdominal obesity & $1.4(0.5-3.7)$ & 0.47 & - & - \\
\hline BMI (per kg/m²) & $1.0(0.9-1.1)$ & 0.38 & - & - \\
\hline Systolic BP (per mm Hg) & $1.00(0.96-1.03)$ & 0.90 & - & - \\
\hline Diastolic BP (per mm Hg) & $1.00(0.95-1.06)$ & 0.98 & - & - \\
\hline Hypoglycemia (severe episodes) & $1.8(0.4-8.6)$ & 0.46 & - & - \\
\hline Smoking & $2.0(0.7-5.9)$ & 0.19 & - & - \\
\hline Physical inactivity & $1.9(0.7-5.6)$ & 0.22 & - & - \\
\hline Continuous subcutaneous insulin infusion & $1.2(0.3-4.3)$ & 0.76 & - & - \\
\hline Antidepressants & $8.3(3.2-21.9)$ & $<0.001$ & $8.4(2.7-26.1)$ & $<0.001$ \\
\hline Lipid lowering drugs & $1.1(0.5-2.4)$ & 0.79 & - & - \\
\hline Antihypertensive drugs & $1.2(0.5-2.6)$ & 0.71 & - & - \\
\hline Cardiovascular complications & $6.6(1.7-25.0)$ & 0.005 & $1.1(0.2-6.3)$ & 0.92 \\
\hline
\end{tabular}

$N=273$; Multiple logistic regression analyses (Backward: Wald); Nagelkerke R Square 0.340; Hosmer and Lemeshow test 0.549 
Table 4 Comparisons between 195 T1DM patients with low and 83 T1DM patients with high levels of sTWEAK

\begin{tabular}{|c|c|c|c|}
\hline & \multicolumn{3}{|l|}{ STWEAK } \\
\hline & Low levels $(<7.2 \mathrm{ng} / \mathrm{ml})$ & High levels ( $\geq 7.2 \mathrm{ng} / \mathrm{ml}$ ) & $P$ - value ${ }^{c}$ \\
\hline$N$ & 195 & 83 & \\
\hline Age (years) & $42(32,51)$ & $42(29,51)$ & 0.79 \\
\hline Diabetes duration (years) & $20(11,30)$ & $18(9,29)$ & 0.58 \\
\hline \multicolumn{4}{|l|}{ Sex } \\
\hline Women & $89(46)$ & $30(36)$ & \multirow[t]{2}{*}{$0.15^{b}$} \\
\hline Men & $106(54)$ & $53(64)$ & \\
\hline Depression & 27 (14) & $2(2)$ & $0.003^{b}$ \\
\hline High galectin-3 $(\geq 2.6 \mathrm{ng} / \mathrm{ml})^{c}$ & $27(14)$ & $14(17)$ & $0.58^{b}$ \\
\hline High HbA1c (> $70 \mathrm{mmol} / \mathrm{mol}(>8.6 \%))$ & $53(27)$ & $22(26)$ & $>0.99^{b}$ \\
\hline Total cholesterol (mmol/l) & $4.6(4.1,5.2)$ & $4.5(4.0,5.0)$ & 0.13 \\
\hline $\mathrm{LDL}(\mathrm{mmol} / \mathrm{l})$ & $2.9(2.4,3.4)$ & $2.8(2.4,3.2)$ & 0.34 \\
\hline Triglycerides (mmol/l) & $0.9(0.7,1.3)$ & $0.8(0.7,1.2)$ & 0.19 \\
\hline $\mathrm{HDL}(\mathrm{mmol} / \mathrm{l})$ & $1.5(1.3,1.8)$ & $1.6(1.3,1.8)$ & 0.71 \\
\hline Triglycerides/HDL-cholesterol ratio & $0.6(0.4-0.9)$ & $0.6(0.4-0.8)$ & 0.23 \\
\hline S-Creatinine $(\mu \mathrm{mol} / \mathrm{l})^{d}$ & $70(62,77)$ & $70(62,80)$ & 0.44 \\
\hline Abdominal obesity ${ }^{e}$ & $33(17)$ & $11(13)$ & $0.48^{b}$ \\
\hline BMI $\left(\mathrm{kg} / \mathrm{m}^{2}\right)^{\mathrm{f}}$ & $24.6(22.4,27.9)$ & $24.6(23.1,26.1)$ & 0.92 \\
\hline Systolic BP (mm Hg) & $120(110,130)$ & $120(110,130)$ & 0.34 \\
\hline Diastolic BP (mm Hg) & $70(70,75)$ & $70(65,78)$ & 0.54 \\
\hline Hypoglycemia (severe episodes) & $7(4)$ & $4(5)$ & $0.74^{b}$ \\
\hline Smoking ${ }^{9}$ & $20(11)$ & $8(10)$ & $>0.99^{b}$ \\
\hline Physical inactivity ${ }^{\mathrm{h}}$ & $24(13)$ & $5(6)$ & $0.19^{b}$ \\
\hline Continuous subcutaneous insulin infusion & $11(6)$ & $14(17)$ & $0.005^{b}$ \\
\hline Antidepressants & $16(8)$ & $6(7)$ & $>0.99^{b}$ \\
\hline Lipid lowering drugs & $92(47)$ & $36(43)$ & $0.60^{b}$ \\
\hline Antihypertensive drugs & $62(32)$ & $26(31)$ & $>0.99^{b}$ \\
\hline Cardiovascular complications & $8(4)$ & $2(2)$ & $0.73^{b}$ \\
\hline
\end{tabular}

$N=278$. Data are presented as median $\left(q_{1}, q_{3}\right)$ or $N(\%) .{ }^{a}$ Mann-Whitney $\mathrm{U}$ test unless otherwise indicated

${ }^{\mathrm{b}}$ Fisher's Exact Test. Missing values: ${ }^{\mathrm{c}} 4 ;^{\mathrm{d}} 12 ;{ }^{\mathrm{e}} 6 ;{ }^{\mathrm{f}} 2 ;{ }^{\mathrm{g}} \mathrm{h}$ h 16

sTWEAK levels [20]. Both depressive states and manic episodes may be symptoms of brain inflammation. Our findings of low sTWEAK levels in the depressed patients imply activation of TWEAK, which has proinflammatory effects on the astrocytes in the brain, which have been demonstrated in vitro [15]. According to previous research, galectin-3 may contribute to microglia activation with sustained inflammatory responses in the brain [26]. These findings are potentially very important as it has been demonstrated in previous research that both astrocytes and microglia are involved in the development of depression [46, 47].

There are several subjects for further research. As this is a cross-sectional study, we can't clarify whether the depressed state leads to immunological disturbances, or if these immunological disturbances lead to a depressed state. To answer this question, it will be necessary to perform longitudinal studies. We will perform a followup exploring the impact of sTWEAK and galectin-3 on cardiovascular complications, comparing with conventional diabetes related risk factors. According to previous research, some antidepressants attenuate immunoinflammatory changes [9]. Whether antidepressants may have impact on the levels of sTWEAK and galectin-3 is a subject for further exploration. As immunoinflammatory changes and the activation of the hypothalamic pituitary axis are part of the stress response, which is involved in depression, it would also be interesting to explore associations between sTWEAK, galectin-3 and cortisol secretion [9]. To compare TWEAK levels between users of MDII and users of CSII in a future larger study would be very interesting. If our 
Table 5 Variables associated with low sTWEAK levels

\begin{tabular}{|c|c|c|c|c|}
\hline & \multicolumn{4}{|c|}{ Low sTWEAK $(<7.2 \mathrm{ng} / \mathrm{ml})$} \\
\hline & COR $(95 \% \mathrm{Cl})$ & $P$ - value & AOR $(95 \% \mathrm{Cl})$ & $\begin{array}{l}P \text { - } \\
\text { value }\end{array}$ \\
\hline Age (per year) & $1.00(0.98-1.03)$ & 0.73 & - & - \\
\hline Diabetes duration (per year) & $1.00(0.98-1.02)$ & 0.71 & - & - \\
\hline Sex (women) & $1.5(0.9-2.5)$ & 0.14 & - & - \\
\hline Depression & $6.5(1.5-28.0)$ & 0.012 & $7.1(1.6-31.4)$ & 0.009 \\
\hline High galectin-3 ( $\geq 2.6 \mathrm{ng} / \mathrm{ml})$ & $0.8(0.4-1.6)$ & 0.49 & - & - \\
\hline High HbA1c (> $70 \mathrm{mmol} / \mathrm{mol}(>8.6 \%))$ & $1.0(0.6-1.8)$ & 0.91 & - & - \\
\hline Total cholesterol (mmol/l) & $1.2(0.9-1.6)$ & 0.19 & - & - \\
\hline $\mathrm{LDL}(\mathrm{mmol} / \mathrm{l})$ & $1.2(0.9-1.7)$ & 0.29 & - & - \\
\hline Triglycerides (mmol/l) & $1.3(0.9-2.0)$ & 0.18 & - & - \\
\hline $\mathrm{HDL}(\mathrm{mmol} / \mathrm{l})$ & $0.8(0.4-1.7)$ & 0.60 & - & - \\
\hline Triglycerides/HDL-cholesterol ratio & $1.5(0.9-2.6)$ & 0.11 & - & - \\
\hline S-Creatinine $(\mu \mathrm{mol} / \mathrm{l})$ & $1.00(0.98-1.01)$ & 0.63 & - & - \\
\hline Abdominal obesity & $1.4(0.6-2.8)$ & 0.42 & - & - \\
\hline BMI $($ per kg/m²) & $0.7(0.4-1.3)$ & 0.26 & - & - \\
\hline Systolic BP (mm Hg) & $1.01(0.99-1.03)$ & 0.33 & - & - \\
\hline Diastolic BP (mm Hg) & $1.00(0.96-1.03)$ & 0.63 & - & - \\
\hline Hypoglycemia (severe episodes) & $0.7(0.2-2.6)$ & 0.64 & - & - \\
\hline Smoking & $1.0(0.4-2.5)$ & 0.92 & - & - \\
\hline Physical inactivity & $2.1(0.8-5.9)$ & 0.14 & - & - \\
\hline Continuous subcutaneous insulin infusion & $0.3(0.1-0.7)$ & 0.004 & $0.3(0.1-0.6)$ & 0.003 \\
\hline Antidepressants users & $1.1(0.4-3.0)$ & 0.78 & - & - \\
\hline Lipid lowering drugs & $1.2(0.7-2.0)$ & 0.56 & - & - \\
\hline Antihypertensive drugs & $1.0(0.6-1.8)$ & 0.94 & - & - \\
\hline Cardiovascular complications & $1.7(0.4-8.3)$ & 0.49 & - & - \\
\hline
\end{tabular}

$N=278$; Multiple logistic regression analyses (Backward: Wald); Nagelkerke R Square: 0.094; Hosmer and Lemeshow test 0.802

findings would be confirmed in a larger study, it would be a very important finding supporting the choice of CSII over MDII. Finally, development of novel therapeutics against the TWEAK/Fn14 axis may be of value both for the treatment or prevention of depression and cardiovascular disease.

Strengths of the study are that inclusion and exclusion criteria were well defined. No patients using systemic corticosteroids, or specific drugs for psychotic or bipolar disorders were included. The findings of this study are new as exploration of the association between STWEAK and depression has not previously been performed in a clinically well-defined setting of T1DM. Neither has the mode of insulin distribution been explored against sTWEAK. The results were controlled for relevant variables which in previous research have been linked to either depression or cardiovascular complications, or to both. The logistic regression models were elaborated for the associations, and calibrated and validated for goodness of fit with the data variables. Precise ELISA techniques were used, and the assays showed low intra-assay coefficients of variation for both sTWEAK and galectin-3. One weakness was that there was no control group with persons without diabetes. Another weakness was that there were very few patients with cardiovascular complications, so no further explorations of associations with cardiovascular complications could be performed. A third weakness was that depression was not assessed by a clinical interview. However, the association between the use of antidepressants and selfreported depression in this study was high, indicating that depression assessed by HADS-D had clinical significance.

\section{Conclusions}

The hypothesis that the depressed patients with T1DM had lower levels of sTWEAK than the non-depressed was confirmed. Low levels of sTWEAK and HDL-cholesterol and high levels of galectin-3 were independently associated with depression in T1DM. These disturbances have previously been associated with cardiovascular disease and mortality, and might contribute to the increased risk for 
cardiovascular disease and mortality previously demonstrated in T1DM patients with depression. We also found that the users of CSII had lower prevalence of low sTWEAK levels than the users of MDII.

\begin{abstract}
Abbreviations
AOR: Adjusted Odds Ratio; BMI: Body Mass Index; Cl: Confidence interval; COR: Crude Odds Ratio; CSII: Continuous subcutaneous insulin infusion; HADS-D: Hospital Anxiety and Depression-Depression subscale; MDII: Multiple daily insulin injections; sTWEAK: Soluble tumour necrosis factor-like weak inducer of apoptosis; T1DM: Type 1 diabetes mellitus; T2DM: Type 2 diabetes mellitus; TNF-receptor: tumour necrosis factorreceptor; WC: Waist circumference
\end{abstract}

\section{Acknowledgements}

The authors are grateful to Anna Lindgren, PhD at the Department of Mathematical Statistics, Lund University, Lund, Sweden, for her statistical skills.

\section{Authors' contributions}

EOM, JD and MH participated as investigators and reviewed, edited, and approved of the final version of the manuscript. EOM initiated the studies of depression in T1DM, performed the statistical analysis, wrote the manuscript, and is the guarantor of this work and, as such, had full access to all the data in the study and takes responsibility for the integrity of the data and the accuracy of the data analysis. MH and JD contributed with their profound knowledge of inflammatory variables and suggested that sTWEAK and galectin-3 would be interesting to explore in this context, and were responsible for the laboratory analyses of sTWEAK and galectin-3.

\section{Authors' information}

EOM is PhD, medical doctor, specialist in Paediatrics and Family Medicine. EOM works at the Department of Research and Development, Region Kronoberg, Växjö, and is affiliated to the Diabetes Research Laboratory, Lund University, Lund. MH is PhD, Associate Professor in biomedicine, and works at the Diabetes Research Laboratory, Lund University, Lund. JD is PhD in biomedicine, and works at the Diabetes Research Laboratory, Lund University, Lund. All Sweden.

\section{Funding}

This research was supported by the Research and Development Fund of Region Kronoberg, Växjö, Sweden, and by the Research Council of South Eastern Sweden (FORSS), Linköping, Sweden. The funding sources were not involved in the collection, analysis and interpretation of data, in the writing of the report, or in the decision to submit the article for publication. Open Access funding provided by Lund University.

\section{Availability of data and materials}

All data are saved at SPSS files for at least 15 years at the Department for Research and Development, Region Kronoberg, Växjö, Sweden. The data sets are not publicly available as individual privacy could be compromised. The data set is available from the corresponding author upon reasonable request.

\section{Ethics approval and consent to participate}

The study was performed in accordance with the Declaration of Helsinki, and was approved by the Regional Ethical Review Board of Linköping University, Linköping (Registration no. M120-07, T89-08). All participants provided written informed consent.

\section{Consent for publication}

Not applicable.

\section{Competing interests}

The authors declare that they have no competing interests.
Received: 17 December 2019 Accepted: 18 November 2020

Published online: 01 December 2020

\section{References}

1. Katsarou A, Gudbjörnsdottir S, Rawshani A, Dabelea D, Bonifacio E, Anderson BJ, et al. Type 1 diabetes mellitus. Nat Rev Dis Primer. 2017;3: 17016.

2. Chillarón JJ, Le-Roux JAF, Benaiges D, Pedro-Botet J. Type 1 diabetes, metabolic syndrome and cardiovascular risk. Metabolism. 2014;63. https:// doi.org/10.1016/j.metabol.2013.10.002.

3. Frostegård J. Immunity, atherosclerosis and cardiovascular disease. BMC Med. 2013;11:1-13.

4. de Groot M, Anderson R, Freedland KE, Clouse RE, Lustman PJ. Association of depression and diabetes complications: a meta-analysis. Psychosom Med. 2001;63:619-30

5. Egede LE, Nietert PJ, Zheng D. Depression and all-cause and coronary heart disease mortality among adults with and without diabetes. Diabetes Care. 2005;28:1339-45.

6. Penninx BW, Milaneschi Y, Lamers F, Vogelzangs N. Understanding the somatic consequences of depression: biological mechanisms and the role of depression symptom profile. BMC Med. 2013;11:129.

7. Korczak DJ, Pereira S, Koulajian K, Matejcek A, Giacca A. Type 1 diabetes mellitus and major depressive disorder: evidence for a biological link. Diabetologia. 2011;54:2483-93. https://doi.org/10.1007/s00125-011-2240-3.

8. Köhler C, Freitas T, de Maes M, De Andrade N, Liu C, Fernandes B, et al. Peripheral cytokine and chemokine alterations in depression: a metaanalysis of 82 studies. Acta Psychiatr Scand. 2017;135:373-87.

9. Leonard BE. Inflammation and depression: a causal or coincidental link to the pathophysiology? Acta Neuropsychiatr. 2018:30:1-16.

10. Al-Fadhel SZ, Al-Hakeim HK, Al-Dujaili AH, Maes M. IL-10 is associated with increased mu-opioid receptor levels in major depressive disorder. Eur Psychiatry. 2019;57:46-51.

11. Chicheportiche Y, Bourdon PR, Xu H, Hsu Y-M, Scott H, Hession C, et al. TWEAK, a new secreted ligand in the tumor necrosis factor family that weakly induces apoptosis. J Biol Chem. 1997;272:32401-10. https://doi.org/ 10.1074/jbc.272.51.32401.

12. Blanco-Colio LM. TWEAK/Fn14 axis: a promising target for the treatment of cardiovascular diseases. Front Immunol. 2014;5:3.

13. Itter A, Orem C, Yucesan FB, Sahin M, Hosoglu Y, Kurumahmutoglu E, et al. Evaluation of serum sTWEAK and SCD163 levels in patients with acute and chronic coronary artery disease. Int J Clin Exp Med. 2015;8:9394.

14. Urbonaviciene G, Martin-Ventura JL, Lindholt JS, Urbonavicius S, Moreno JA, Egido J, et al. Impact of soluble TWEAK and CD163/TWEAK ratio on longterm cardiovascular mortality in patients with peripheral arterial disease. Atherosclerosis. 2011;219:892-9.

15. Saas P, Boucraut J, Walker PR, Quiquerez A, Billot M, Desplat-Jego S, et al. TWEAK stimulation of astrocytes and the proinflammatory consequences. Glia. 2000;32:102-7.

16. Llaurado G, Gonzalez-Clemente J-M, Maymo-Masip E, Subías D, Vendrell J, Chacon MR. Serum levels of TWEAK and scavenger receptor CD163 in type 1 diabetes mellitus: relationship with cardiovascular risk factors. A casecontrol study. PLoS One. 2012;7:e43919.

17. Kralisch S, Ziegelmeier M, Bachmann A, Seeger J, Lössner U, Blüher M, et al. Serum levels of the atherosclerosis biomarker sTWEAK are decreased in type 2 diabetes and end-stage renal disease. Atherosclerosis. 2008;199:440-4. https://doi.org/10.1016/j.atherosclerosis.2007.10.022.

18. Dereke J, Nilsson J, Nilsson C, Strevens H, Landin-Olsson M, Hillman M. Soluble CD163 and TWEAK in early pregnancy gestational diabetes and later glucose intolerance. PLoS One. 2019;14:e0216728.

19. Schmidt FM, Koch J, Nowak C, Holdt LM, Teupser D, Hegerl U, et al. Ligands and receptors of the TNF superfamily are decreased in major depression and during early antidepressant therapy. J Psychiatr Res. 2019;119:116-21.

20. Yirün MC, Yirün O, Ünal K, Yüksel RN, Altunsoy N, Yaylaci ET, et al. Serum TNF-related weak inducer of apoptosis (TWEAK) and TNF-related apoptosisinducing ligand (TRAIL) levels of patients with bipolar disorder in manic episode, in remission and healthy controls. Psychiatry Res. 2017;257:338-45.

21. Henderson NC, Sethi T. The regulation of inflammation by galectin-3. Immunol Rev. 2009;230:160-71.

22. Kusaka H, Yamamoto E, Hirata Y, Fujisue K, Tokitsu T, Sugamura K, et al. Clinical significance of plasma galectin-3 in patients with coronary artery disease. Int J Cardiol. 2015;201:532-4. 
23. Kang Q, Li X, Yang M, Fernando T, Wan Z. Galectin-3 in patients with coronary heart disease and atrial fibrillation. Clin Chim Acta. 2018;478:166-70.

24. Gehlken C, Suthahar N, Meijers WC, de Boer RA. Galectin-3 in heart failure: an update of the last 3 years. Biomark Heart Fail. 2018;14:75-92. https://doi. org/10.1016/j.hfc.2017.08.009.

25. Imran TF, Shin HJ, Mathenge N, Wang F, Kim B, Joseph J, et al. Meta-analysis of the usefulness of plasma Galectin-3 to predict the risk of mortality in patients with heart failure and in the general population. Am J Cardiol. 2017;119:57-64. https://doi.org/10.1016/j.amjcard.2016.09.019.

26. Burguillos MA, Svensson M, Schulte T, Boza-Serrano A, Garcia-Quintanilla A Kavanagh E, et al. Microglia-secreted galectin-3 acts as a toll-like receptor 4 ligand and contributes to microglial activation. Cell Rep. 2015;10:1626-38.

27. Gagno G, Padoan L, Stenner E, Beleù A, Ziberna F, Hiche C, et al. Galectin 3 and galectin 3 binding protein improve the risk stratification after myocardial infarction. J Clin Med. 2019;8:570

28. Feingold KR, Grunfeld C. Effect of inflammation on HDL structure and function. Curr Opin Lipidol. 2016;27:521-30.

29. Melin EO, Dereke J, Hillman M. Female sex, high soluble CD163, and low HDL-cholesterol were associated with high galectin-3 binding protein in type 1 diabetes. Biol Sex Differ. 2019;10:51. https://doi.org/10.1186/s13293019-0268-0.

30. Lind M, Svensson A-M, Kosiborod M, Gudbjörnsdottir S, Pivodic A, Wedel H, et al. Glycemic control and excess mortality in type 1 diabetes. N Engl J Med. 2014;371:1972-82.

31. Melin EO, Thunander M, Svensson R, Landin-Olsson M, Thulesius HO. Depression, obesity and smoking were independently associated with inadequate glycemic control in patients with type 1 diabetes. Eur J Endocrinol. 2013;168:861-9.

32. Melin EO, Thunander M, Landin-Olsson M, Hillman M, Thulesius HO Depression, smoking, physical inactivity and season independently associated with midnight salivary cortisol in type 1 diabetes. BMC Endocr Disord. 2014;14:75.

33. Melin EO, Dereke J, Thunander M, Hillman M. Depression in type 1 diabetes was associated with high levels of circulating galectin-3. Endocr Connect. 2018;7:819-28.

34. Melin EO, Thulesius HO, Hillman M, Svensson R, Landin-Olsson M, Thunander M. Lower HDL-cholesterol, a known marker of cardiovascular risk, was associated with depression in type 1 diabetes: a cross sectional study. Lipids Health Dis. 2019;18:65. https://doi.org/10.1186/s12944-0191009-4

35. Melin EO, Dereke J, Thunander M, Hillman M. Soluble CD163 was linked to galectin-3, diabetic retinopathy and antidepressants in type 1 diabetes. Endocr Connect. 2018;7. doi.org/https://doi.org/10.1186/s13293-019-0268-0.

36. Melin EO, Dereke J, Hillman M. Higher levels of the soluble receptor for advanced glycation end products and lower levels of the extracellular newly identified receptor for advanced glycation end products were associated with lipid-lowering drugs in patients with type 1 diabetes: a comparative cross-sectional study. Lipids Health Dis. 2020;19:1-10.

37. Melin EO, Svensson R, Gustavsson S- $\AA$, Winberg A, Denward-Olah E, LandinOlsson $M$, et al. Affect school and script analysis versus basic body awareness therapy in the treatment of psychological symptoms in patients with diabetes and high $\mathrm{HbA1c}$ concentrations: two study protocols for two randomized controlled trials. Trials. 2016;17 2016;17:221:221. doi:https://doi. org/10.1186/s13063-016-1347-8.

38. Zigmond AS, Snaith RP. The hospital anxiety and depression scale. Acta Psychiatr Scand. 1983;67:361-70. https://doi.org/10.1111/j.1600-0447.1983. tb09716.x.

39. Bocéréan C, Dupret E. A validation study of the hospital anxiety and depression scale (HADS) in a large sample of French employees. BMC Psychiatry. 2014;14:1-11. https://doi.org/10.1186/s12888-014-0354-0.

40. Klein S, Allison DB, Heymsfield SB, Kelley DE, Leibel RL, Nonas C, et al. Waist circumference and Cardiometabolic risk: a consensus statement from shaping America's health: Association for Weight Management and Obesity Prevention; NAASO, the Obesity Society; the American Society for Nutrition; and the American Diabetes Association. Obesity. 2007;15:1061-7. https://doi. org/10.1038/oby.2007.632.

41. Uruska A, Zozulinska-Ziolkiewicz D, Niedzwiecki P, Pietrzak M, WieruszWysocka $\mathrm{B}$. TG/HDL-C ratio and visceral adiposity index may be useful in assessment of insulin resistance in adults with type 1 diabetes in clinical practice. J Clin Lipidol. 2018;12:734-40.
42. The National Board of Health and Welfare. Swedish National Guidelines for Diabetes. 2009. https:/www.socialstyrelsen.se. Accessed 13 March 2009.

43. Ranganathan P, Pramesh CS, Aggarwal R. Common pitfalls in statistical analysis: logistic regression. Perspect Clin Res. 2017;8:148-51. https://doi.org/ 10.4103/picr.PICR_87_17.

44. Rader DJ, Hovingh GK. HDL and cardiovascular disease. Lancet. 2014;384: 618-25

45. Zou XH, Sun LH, Yang W, Li BJ, Cui RJ. Potential role of insulin on the pathogenesis of depression. Cell Prolif. 2020;53:e12806.

46. Peng L, Verkhratsky A, Gu L, Li B. Targeting astrocytes in major depression. Expert Rev Neurother. 2015;15:1299-306. https://doi.org/10.1586/14737175. 2015.1095094.

47. Brites D, Fernandes A. Neuroinflammation and depression: microglia activation, extracellular microvesicles and microRNA dysregulation. Front Cell Neurosci 2015;9:476. https://doi.org/https://doi.org/10.3389/fncel.2015. 00476.s.

\section{Publisher's Note}

Springer Nature remains neutral with regard to jurisdictional claims in published maps and institutional affiliations.
Ready to submit your research? Choose BMC and benefit from:

- fast, convenient online submission

- thorough peer review by experienced researchers in your field

- rapid publication on acceptance

- support for research data, including large and complex data types

- gold Open Access which fosters wider collaboration and increased citations

- maximum visibility for your research: over $100 \mathrm{M}$ website views per year

At BMC, research is always in progress.

Learn more biomedcentral.com/submissions 